Copyright ( 2013 IEEE. Personal use of this material is permitted. Permission from IEEE must be obtained for all other uses, in any current or future media, including reprinting/republishing this material for advertising or promotional purposes, creating new collective works, for resale or redistribution to servers or lists, or reuse of any copyrighted component of this work in other works. 


\section{Exhaust Plume Flow Visualization for Qualitative Analysis of Engine Combustion Performance}

\author{
Masood Mehmood Khan \\ Faculty of Science \& Engineering \\ Curtin University, Perth Western Australia \\ Masood.Khan@curtin.edu.au
}

\author{
Hammad Iqbal Sherazi \\ National University of Science \& Technology \\ PNEC Karachi \\ H.sherazi@pnec.edu.pk
}

\begin{abstract}
This work explores use of Thermal Infrared Image based Flow Visualization (TIIFV) for qualitative analysis of gasoline engine combustion performance. It proposes determining engine combustion performance through analysis of the exhaust plume turbulence and radiation extinction patterns. The employed methodology requires estimating the point spread function (PSF) prevailing in a LWIR image and using the PSF estimates for enhancing the engine exhaust plume LWIR images. Influence of exhaust plume composition on the plume flow characteristics, made evident by the turbulence and radiation extinction patterns, is then ascertained. The observed plume flow characteristics and underlying flow patterns are used to qualitatively determine the engine combustion performance. Results suggest that engine exhaust flow visualization can help in qualitative analysis of combustion performance from a distance and our reliance on photochemical-based analysis of gasoline engine combustion efficiency can be reduced. Thus a time consuming and untidy process, difficult to be carried out in real life situations, may be replaced with a swift and cleaner one.
\end{abstract}

Keywords-longwave thermal infrared imaging, engine combustion performance analysis, exhaust plume flow visualization, vehicle comustion analysis.

\section{INTRODUCTION}

Gasoline engine (GE) exhaust gases may contain some 200500 different species [1]. The amount and proportions of prevailing species depend on; the quality of combustion, the kind and quantities of additives used in a gasoline compound, and the types of primary secondary and tertiary chemical reactions caused by the GE exhaust particles released in the atmosphere.

The gross weight related calculations are used in collecting GE exhaust emission data. As the GE exhaust particles vary in size and weight, the typically acquired emission data won't allow distinguishing between the emission characteristics of two equal weight GE exhaust samples. In real life, each sample would carry different amounts (and proportions) of larger and smaller particles. Furthermore, it is known that equal weights of gases carrying varying quantities of smaller and larger particles, when released to the atmosphere, would exhibit differences in atmospheric residence time, local visibility and cloud-nucleation [2]. Factors like chemical composition, nature of involved chemical reactions, and size distribution of particles influence exhaust gases' atmospheric residence time. Also, in addition to these factors, discharged particles' refractive indices affect visibility and influences cloud-nucleation [2,3].

Analyzing gasoline engine (GE) combustion efficiency using the photochemical-based method, though common, is a time consuming process that needs physical access to the vehicle under scrutiny. An ability to swiftly perform qualitative GE combustion analysis, from a distance, would therefore help. One way of performing qualitative combustion analysis from a distance is to establish the relationship between the compositions of GE exhaust gases, resulting local atmospheric visibility and atmospheric residence time. This approach could be easily implemented through visualization and examination of the GE exhaust flow characteristics. Thermal InfraRed Imaging (TIRI) would help in visualizing both thermal and visible aspects of the fluid flow. Major GE exhaust plume species can be visualized in LongWave InfraRed (LWIR) band. Thus TIRI, in LongWave InfraRed (LWIR) band, makes it possible to qualitatively determine the combustion performance of GE exhaust through examination of the observed thermal disturbance patterns.

Previous works suggest that vehicle engine combustion analysis from a distance is possible; can be useful in real life situations; and can be facilitated in LWIR band [4,5]. Building upon the previous works, this paper exploits thermal infrared image-based flow visualization (TIIFV) for examining the thermal disturbance patters in GE exhaust plume and interpreting the GE combustion performance in qualitative terms.

In section II, engine exhaust visualization is introduced. Section III introduces the gasoline engine exhaust in Infrared spectrum. The experimental design used for this investigation is reported in Section IV. The employed methodology including the point spread function estimation, and noise and blur reduction techniques are reported in Section V. The isotherm detection techniques for discovering thermal disturbances in GE exhaust plumes' LWIR images are also reported in Section V. Results are reported in Section VI.

\section{GASOLINE ENGINE EXHAUST IN LWIR SPECTRUM}

Combustion products discharged from a GE to the atmosphere exhibit an unpredictable flow pattern for the following reasons. The transported material quantities are mixed at a rate faster than the mixing rate of gases undergoing only the molecular diffusion processes. The exhaust products span over a wide range of spatial wavelengths. The exhaust 


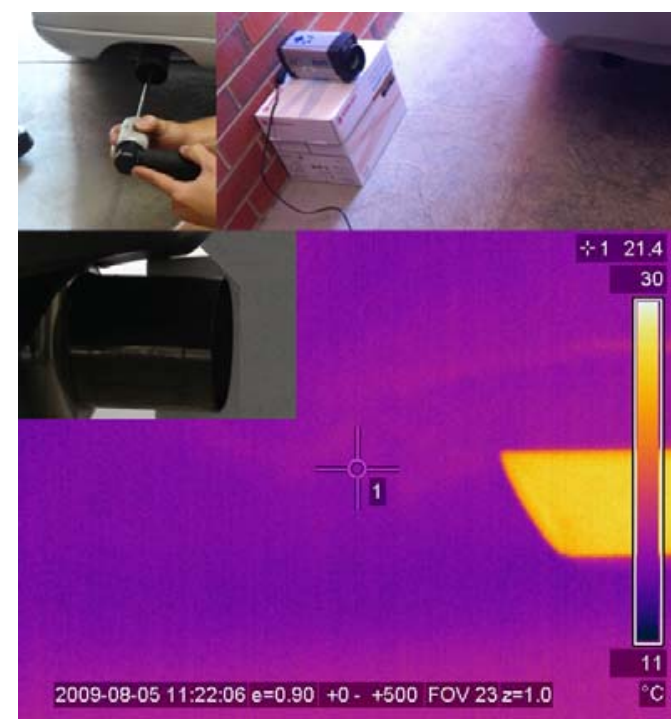

Fig. 1. Top left- combustion analysis; Top right- thermal infrared imaging set up, Bottom left- polystyrene foam sheet behind the exhaust mufler; Bottom-L WIR image of exhaust plume.

flow has a non-zero vorticity in multiple regions, which causes the upstream flow to gradually become irrotational. These flow features make the GE combustion exhaust flow highgradient, unsteady, and turbulent $[1,11,12,13]$.

Typical GE combustion products contain carbon dioxide $\left(\mathrm{CO}_{2}\right)$, water vapors $\left(\mathrm{H}_{2} \mathrm{O}\right)$, Nitrogen $\left(\mathrm{N}_{2}\right)$ oxides of nitrogen (NOX), carbon monoxide (CO), unburned hydrocarbons, partially oxidized hydrocarbons, sulfur dioxide $\left(\mathrm{SO}_{2}\right)$, particulates and smoke $[6,14,15]$.

All thermal radiations are detectable in 0.1-100 $\mu \mathrm{m}$ wavelength [16] thus radiations from a majority of the GE exhaust species can be detected in LWIR band. The volumetric and stimulated emissions of several aromatic hydrocarbons $(\mathrm{AH})$ produced during the gasoline combustion are also detectable in the long-wave infrared (LWIR) spectrum (8-14 $\mu \mathrm{m})$. The volumetric and stimulated emissions of $\mathrm{CO}$, $\mathrm{CO}_{2}$ and other non-methane hydrocarbons such as Ethylbenzene $\left(\mathrm{C}_{8} \mathrm{H}_{10}\right)$, 2-Butene $\left(\mathrm{C}_{4} \mathrm{H}_{8}\right)$, and 2-pentene $\left(\mathrm{C}_{5} \mathrm{H}_{10}\right)$ can also be detected in midwave infrared cameras.

\section{Visualizing Engine Exhaust Plume}

Flow visualization methods are typically applied to visible spectrum images and mathematically generated images. The later involve computational fluid dynamics (CFD). Common flow visualization approaches attempt to determine the flow characteristics and estimate species concentration, mostly through excitation of specific species fluorescence [14]. Typically, optical imaging tools and methods focus on in-situ testing of combustion performance by analyzing the exhaust flow in laboratory conditions. In the recent past, flow visualization techniques have been used for remotely analyzing the GE combustion performance [15-18].

Image-based flow visualization (IBFV) techniques such as
TABLE I

COMbustion ANALysis RESUltS OF THREE UNLEADEDPETROLEUM RUN ENGINES

\begin{tabular}{|c|c|c|c|c|c|c|c|}
\hline 窇. & 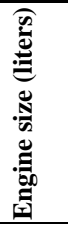 & $\begin{array}{l}\widehat{\Xi} \\
\hat{\bar{\Xi}} \\
0 \\
0\end{array}$ & 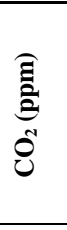 & 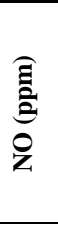 & 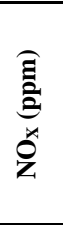 & 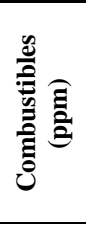 & 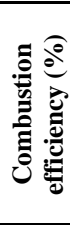 \\
\hline 1. Impreza & 1.80 & 0 & 11.9 & 84 & 127 & 0.3 & 84.3 \\
\hline 2. Corolla & 1.79 & 0 & 11.9 & 121 & 10 & 0.1 & 85.3 \\
\hline 3. Yaris & 1.29 & 0 & 12 & 10 & 0 & 0.3 & 85.2 \\
\hline
\end{tabular}

the one proposed in [19] provide a framework for visualizing moving particle flow, streamline patterns and moving textures. The IBFV techniques are typically used for analyzing visible spectrum and CFD generated images. The IBFV methods were also employed for extracting features of typical high-gradient, compressible turbulent flow [18]. In this work, the IBFV approach was extended and developed for visualizing LWIR images. We refer to this approach as Thermal Infrared Image based Flow Visualization (TIIFV)

\section{EXPERIMENTAL SETUP}

Having realized the feasibility of using LWIR for GE exhaust plume imaging, first the engine combustion efficiencies of several randomly picked cars were analyzed. Results of the three of these unleaded petroleum-run vehicles' engines are being discussed in this paper. An electrochemical sensing based combustion analyzer was used for engines' combustion analyses. The percentages of various species and engines' combustion efficiencies are shown in Table I. Figure 1 shows the combustion analysis setup, infrared imaging arrangement and an acquired LWIR image of exhaust plume. The three cars had similar combustion efficiencies and their exhaust gases had similar chemical compositions.

During the image acquisition, the engines were set to run at $2000 \mathrm{rpm}$. A LWIR (8.0-14.0 $\mu \mathrm{m})$ was used for thermal imaging. The camera was set normal to the exhaust stream, about 500 centimeters away. This resulted in a consistent and pre-determined field of view (FoV). The set up was also helpful in minimizing the effects of rapid mixing and dispersion of pollutants in the atmosphere [9]. Walled arrangements were preferred for avoiding any major air drift and maintaining the atmospheric conditions during the image acquisition session. The emissivity contrast between the background and foreground elements of LWIR images was maximized using thermoset polystyrene foam sheet (low emissivity, $\varepsilon \approx 0.50$ ) in the background. Polystyrene foam also has a low thermal conductivity $(0.025 \sim 0.03 \mathrm{~W} / \mathrm{mK})$ [13] so it maintains a near-constant temperature under normal atmospheric conditions. 

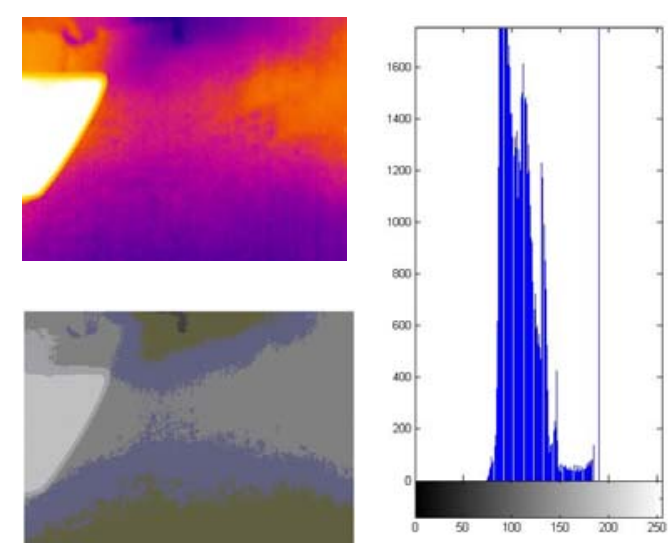

Fig. 2. Top-left-LWIR image; Bbottom-left - Literal separation of thermal radiations emitted by pixels of combustion species in foreground; Rright - grey-level histogram showing separation of background and foreground.

The combustion exhaust plume temperatures added with the cold atmospheric air $\left(\approx 19-21{ }^{\circ} \mathrm{C}\right)$ were not hot sufficient to immediately increase (or decrease) the polystyrene foam sheet temperatures. Figure 2 shows a LWIR image and its grey-level histogram and exhibits that the background and foreground pixels are separable.

\section{COMPUTATIONAL APPROACH}

This section presents the employed image analysis, image enhancement and thermal disturbance pattern analysis methods, illustrated in Figure 3. The image acquisition set up was introduced in previous section.

\section{A. Noise and blur reduction}

Generally, the Gaussian noise model can be assumed to prevail in the LWIR images. With this assumption, even in the absence of a priori knowledge of the point spread, the blind deconvolution method is generally adopted for image restoration [20].

Removing noise from LWIR fluid flow images poses some unique challenges. Firstly, an image degradation model is difficult to predict for the movement of low-density clusters/clouds of radiation for a low contrast factor. Secondly, the multi-dimensional and multi-speed movements of particles and molecules result in added blur for the aberration factor. The problem is accentuated because of the unpredictable nature of high-gradient, unsteady turbulent fluid flow. Thus estimating degradation function $H(x, y)$ caused by the noise $\eta(x, y)$ in a fluid flow LWIR image $g(x, y)$ is a complex task.

This work opted to estimate the point spread function (PSF) and use the estimated PSF with an iterative nonlinear filter for noise reduction and blur reduction. Considering the complex motion and extinction phenomena prevailing in the combustion exhaust plume LWIR images, it was appropriate to assume that a rotationally symmetric PSF would allow for modeling the noise and blur. Thus, the aberration-variance based PSF estimation [21] was employed as

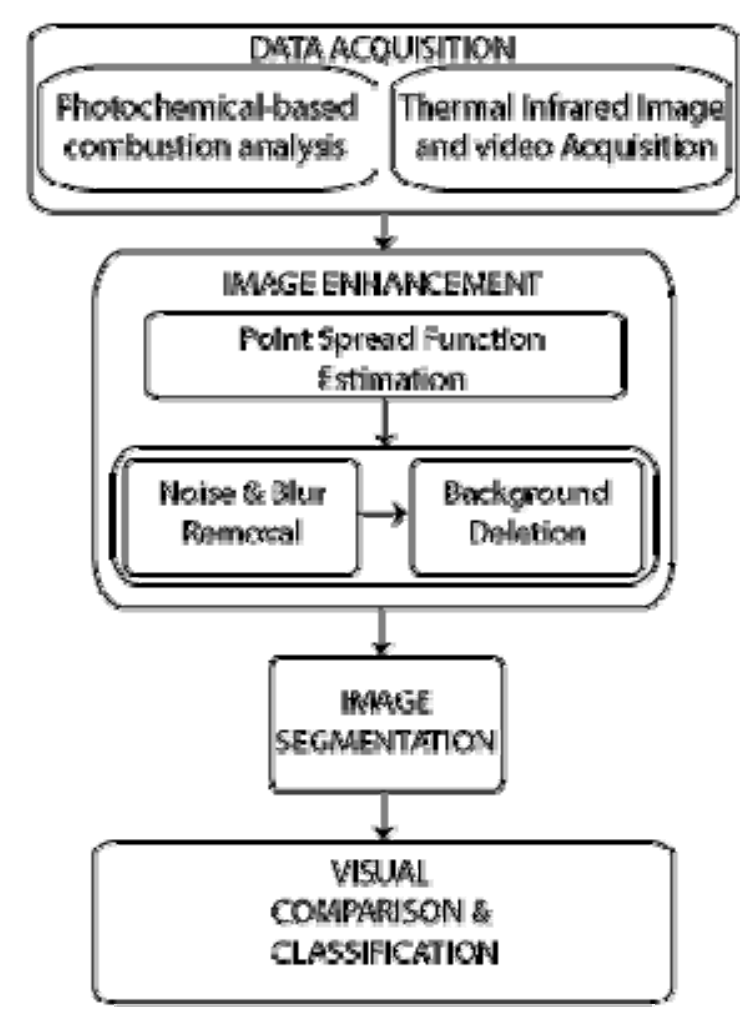

Fig. 3. Employed data acquisition and computational approaches.

$$
\mathrm{I}_{\mathrm{A}}=\mathrm{I}(\mathrm{z}) /\left[2 \mathrm{~J}_{1}(\mathrm{z}) / \mathrm{z}\right]^{2} \text {. }
$$

Equation (1) leads to the generalizations in equations (2) and (3) as:

$$
\begin{aligned}
& \mathrm{I}(\mathrm{z}) \cong \mathrm{S}\left[2 \mathrm{~J}_{1}(\mathrm{z}) / \mathrm{z}\right]^{2} \exp \left(-\mathrm{k}^{2}\left\{\mathrm{gF}(\mathrm{z})-[\mathrm{F}(\mathrm{z})]^{2}\right\}\right), \\
& \mathrm{F}(\mathrm{z})=\left[1-\left(\alpha_{1} / \mathrm{z}\right)^{2}\right]^{-1}
\end{aligned}
$$

In the above equations, $I(z)$ is the diffraction angle that describes the intensity distribution (or PSF), $z$ is a dimensionless optical coordinate in the image plane and $J_{l}$ is the first-order Bessel function of the first kind. The quantity $k(=2 \pi / \lambda)$ represents the wave number, $S$ denotes the Strehl ratio, $g$ results from the wave-front moment $(\Delta)^{2}$ and $\alpha$ represents the sampling point in above equations.

The effectiveness of the employed noise reduction approach was validated. The classical Haralick edge detection algorithm [22] was invoked on (1) the LWIR images restored by the aforementioned approach and (2) the LWIR images restored using the Gaussian filter. The aberration-variance based PSF estimation resulted in a much better edge detection in the LWIR images.

The estimated PSF values were used in the frequency domain for invoking the Lucy-Richardson iterative non-linear filter on the LWIR images. This filter modeled LWIR images using Poisson statistics and resulted in an iterative process that continued till the following convergence was not satisfied [23]: 

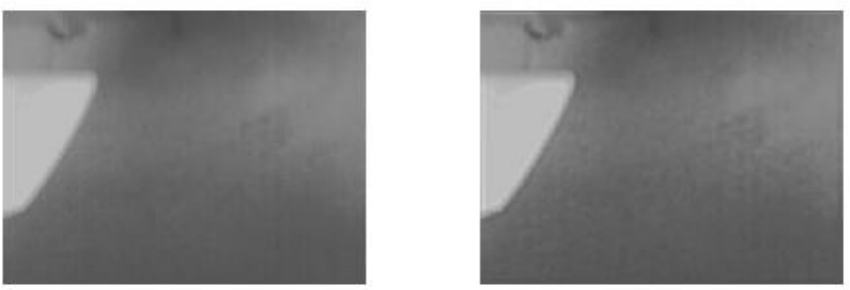

Fig. 4. Separation of thermoset polystyrene foam background and foreground combustion species pixels: (a)-LWIR image; (b)- grey-level histogram.

$$
\hat{\mathrm{f}}_{\mathrm{k}+1}(\mathrm{x}, \mathrm{y})=\hat{\mathrm{f}}_{\mathrm{k}}(\mathrm{x}, \mathrm{y})\left[\mathrm{h}(-\mathrm{x}-\mathrm{y}) \otimes \frac{\mathrm{g}(\mathrm{x}, \mathrm{y})}{\mathrm{h}(\mathrm{x}, \mathrm{y}) \otimes \hat{\mathrm{f}}_{\mathrm{k}}(\mathrm{x}, \mathrm{y})}\right] \text {. }
$$

The symbol $\hat{f}_{k+1}(x, y)$ in equation (4) represents the degradation in the acquired LWIR image $g(x, y)$ of the actual image $f(x, y)$, and $h(x, y)$ is the spatial representation of the degradation function.

This 2-stage noise and blur reduction approach, adopted keeping the GE exhaust LWIR image details and complexities highlighted a higher level of image details.

\section{B. Thermal distrubance visualization through image segmentation}

Thermoset polystyrene has a low thermal conductivity $(0.025 \sim 0.03 \mathrm{~W} / \mathrm{mK})$. Keeping thermoset polystyrene foam sheets in the background (Figure 1), resulted in maintaining higher emissivity and temperature contrasts between the background and the foreground of the LWIR images. It was safe to assume that the foam sheets would maintain an almost constant temperature $\left(T_{f}\right)$ during every instance of image capture. Thus, the thermal distributions in each LWIR image could be realized by comparing the temperature of each region (a group of pixels having similar grey-level values) with the measured $T_{f}$ as:

$$
\Delta \mathrm{T}=\mathrm{T}_{\mathrm{f}}-\mathrm{T}_{\mathrm{pn}} .
$$

$\Delta T$ in equation (5) is the temperature gradient and $T_{p n}$ represents temperature of $\mathrm{n}^{\text {th }}$ plume region in the LWIR image. Such a computational approach would result in a set of distinguishable thermal regions as:

$$
\Delta \mathrm{T}_{\mathrm{j}-1} \neq \Delta \mathrm{T}_{\mathrm{j}} \neq \Delta \mathrm{T}_{\mathrm{j}+1}
$$

Such a thermal separation of regions of GE LWIR images would require invoking an effective image segmentation approach.

Several well tested image segmentation techniques such as edge detection, transformation and thresholding have been employed in fluid visualization. The comparative advantages and limitations of these algorithms in segmenting the LWIR images of turbulent fluid are available in the literature. As highlighted through equations (5) and (6), the region splitting and merging algorithm, based on the temperature and pixel grey-level differences, would divide the GE LWIR into
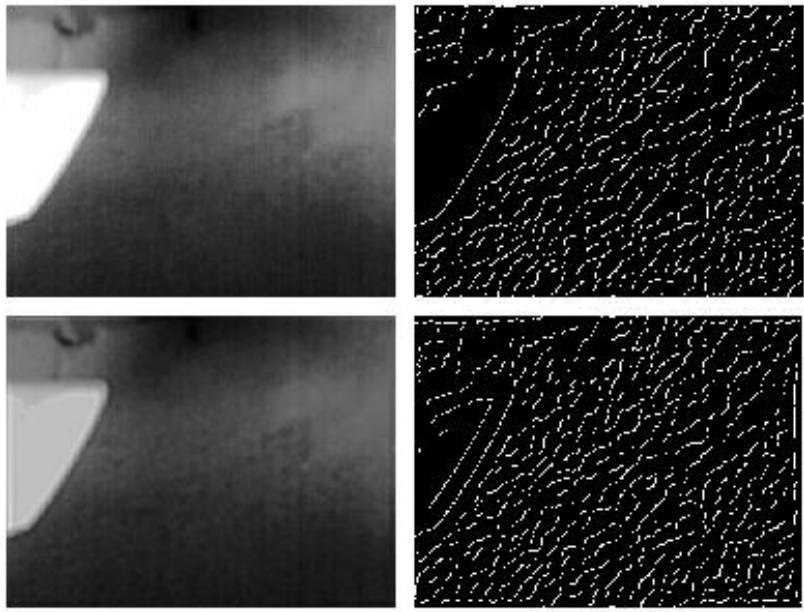

Fig. 5. Top row - Gaussian filter; and Bottom row - The classical Haralick edge detection algorithm invoked after noise and blur removal using the The classical Haralick edge detection algorithm invoked after noise and blur removal using the 2-stage image restoration method.

arbitrary regions. The split-merge process would continue until a set of prescribed conditions are met for the best possible division of the LWIR image into thermally distinguishable regions. The employed split-merge algorithm is given below.

Assuming $L=$ LWIR image, the objective is to partition $L$ into $n$ sub-regions $L_{1}, L_{2}, \ldots, L_{n}$ given the following conditions:

1. Each $\mathrm{L}_{i}$ is a connected region $(\mathrm{i}=1,2,3, \ldots, \mathrm{n})$;

2. $\bigcup_{\mathrm{i}=1}^{\mathrm{n}} \mathrm{L}_{\mathrm{i}}=\mathrm{L}$; for completing the segmentation

3. $\mathrm{L}_{\mathrm{k}} \cap \mathrm{L}_{1}=\phi$; $\Phi$ is a null set such that each ' $i$ ' and $\mathrm{k} \neq 1$;

4. $P\left(L_{i}\right)=$ True; for $i=1,2,3, \ldots, n$, and

5. $\mathrm{P}\left(\mathrm{L}_{\mathrm{i}} \cup \mathrm{L}_{\mathrm{j}}\right)=$ False for $i \neq j$.

\section{RESULTS}

Figure 4 shows noise and blur reduction in a LWIR image using the aforementioned 2-stage image restoration approach. Figure 5 shows the effectiveness of the aberration-variance based PSF estimation method against another widely used image restoration method, the Gaussian filter. It is obvious in Figure 5 that a superior noise and blur reduction approach would result in better image segmentation and edge detection, particularly for visualizing the high-gradient turbulent fluid flow. The three engines' LWIR images exhibited similar thermal disturbance patterns.

The image segmentation results shown in Figure 6 highlight important characteristics of the GE combustion exhaust flow.

The temperature distribution in Figure 6 represents an isotropic turbulence and shows the scale of turbulence varying isotropically. Figure 6 also exhibits growth in the areas of isotherms as fluid particles move away (in both horizontal and vertical directions) and extinct in the atmosphere. These initial 


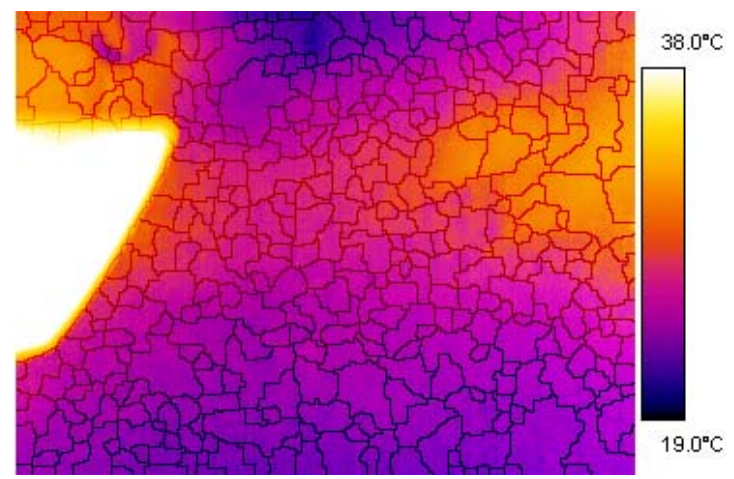

Fig. 6. The thermal distrubance patterns and isotherms superimposed on the actual LWIR image of GICE exhaust flow image. The temperature scale is shown in the right corner.

results highlight the efficacy of the employed algorithmic approach.

Figure 7 compares the LWIR images and segmentation results of the two GE's running at similar combustion efficiencies. In the left column, an Impreza's GE, running at $84.3 \%$ combustion efficiency is shown. The right column of Figure 7 shows a LWIR image and segmentation results of a Toyota Yaris running at $85.2 \%$ combustion efficiency. Though the two GE's had similar combustion efficiencies, the thermal disturbance patterns and isotherm sizes in Figure 7 differ. The high combustion efficiency GE images show smaller isotherms and better isotropic distribution than those obvious in the images of a low combustion efficiency GE. A closer look at the two segmented images in Figure 7 would suggest how the differences in GE efficiencies could result in different isotherm sizes and spatial distribution of thermal patterns.

\section{DISCUSSION AND CONCLUSION}

Fulfilling the condition, $\mathrm{L}_{\mathrm{k}} \cap \mathrm{L}_{1}=\phi$ (recall $\phi$ is a null set for all ' $i$ ' and $k \neq 1$ ) would pose certain limits on the region splitting and merging algorithm. Mainly because of the complex particle and radiation motion, diffusion and extinction phenomena associated with the turbulent fluid flow. This problem could be minimized by selecting proper splitting and merging parameters. Thus an adaptive selection of spilt and merge parameters is recommended. However, transmission of radiation through atmosphere is affected by absorption, scattering and emission. Turbulence, added to these factors, makes it difficult to estimate the true region splitting and merging parameters. The non-continuous nature of thermal extinctions in the turbulent flow LWIR images makes it difficult to identify the isotherms.

Despite these factors, the expected vertical and horizontal thermal asymmetries of turbulent flow were easily visualized through the proposed region split-merge algorithm. These initial results suggest that discovering useful thermal
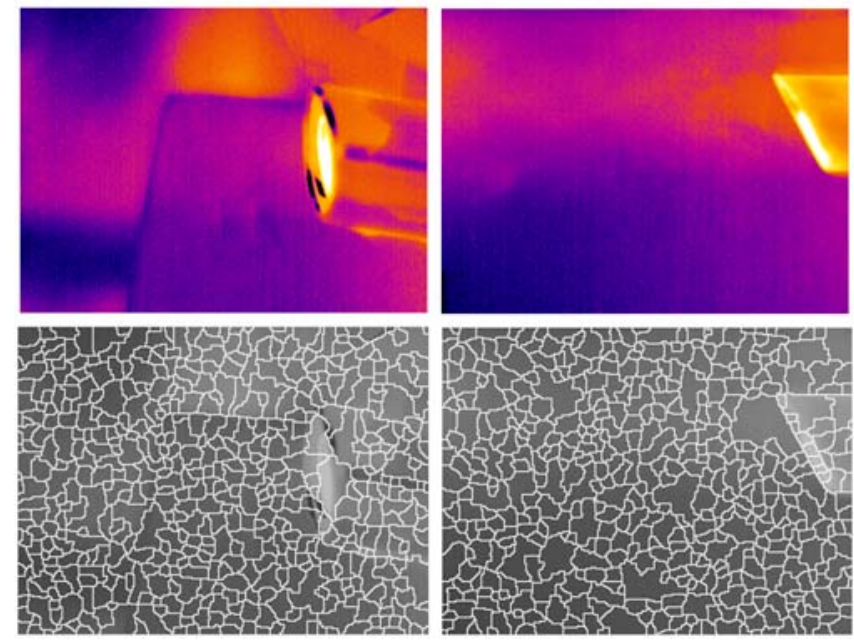

Fig. 7. Left column - Isotherms in $84.3 \%$ combustion efficiency Impreza GE LWIR. Right column - Isotherms in $85.2 \%$ combustion efficiency Yaris GE LWIR).

disturbance patterns in LWIR of combustion exhaust flow images is possible. As demonstrated through this work, a careful image enhancement strategy would allow identifying movement of radiation carrying media particles. The differences in the observed thermal radiation patterns, isotherm sizes and spatial movements can be used to qualitatively distinguish between GE combustion efficiencies. The employed TIIFV approach may allow identifying the bad performing vehicle engines from a distance and may help environmental regulations enforcing agencies in further testing the (identified) poorly performing vehicles. As a result, time and efforts wasted in testing satisfactorily performing vehicles can be saved.

\section{REFERENCES}

[1] Faiz, C. S. Weaver, and M. P. Walsh, Air Pollution from Motor Vehicles. Washington D.C.: The World Bank, 1996.

[2] U.S. Department of SERVICE, N. T. I. \& COMMERCE, 1972. Abatement of particulate emissions from stationary sources. Washington, D.C.: National Academy of Engineering.

[3] R. Siegel and J.R. Howell, Thermal Radiation Heat Transfer, Washington, Hemisphere Publishing Corporation, 1992.

[4] G. Bishop, S. McLaren and D.H. Stedman, Apparatus for remote analysis of vehicle emissions, 1996.

[5] D.H. Stedman and G. Bishop, System and method for remote analysis of small engine vehicle emissions. USA patent application, 2003.

[6] R. S. Cant and Mastorakos, An Introduction to Turbulent Reacting Flows. London: Imperial College Press, 2008.

[7] G. T. Scanady, Turbulent Diffusion in the Environment. Dordrecht, Netherlands: Kluwer Academic Publishing, 1973.

[8] M. Lesieur, Turbulence in Fluids. London: Kluwer Academic Publishers, 1997.

[9] J. Smith and T. T. Lim, Flow Visualization. London: Imperial College Press, 2000.

[10] E. L. Keating, Applied Combustion. New York: Marcel Dekkar Inc., 1993.

[11] E. Baukal Jr., Oxygen Enhanced Combustion. New York: CRC Press, 1998. 
[12] Y. Goswami, F. Kreith, and J. F. Kreider, Principles of Solar Engineering. New York: Taylor \& Francis, 2000.

[13] "Oxford Dictionary of Chemistry," in Oxford Dictionary of Chemistry, 5th ed, J. Daintith, Ed. Oxford: Oxford University Press, 2004.

[14] B. Jahne, Practical Handbook on Image Processing for Scientific Applications. New York: CRC Press, 1997.

[15] J. H. W. Prengle, C. A. Morgan, C.-S. Fang, L.-K. Huang, P. Campani, and W. W. Wu, "Infrared Remote Sensing and Determination of Pollutants in Gas Plumes," Environmental Science and Technology, vol. 7, pp. 417-423, 1973.

[16] M. C. H. Lim, G. A. Ayoko, L. Morawska, Z. D. Ristovski, E. R. Jayaratne, and S. Kokot, "A Comparative Study of the Elemental Composition of the Exhaust Emissions of Cars Powered by Liquified Petroleum Gas and Unleaded Petrol," Atmospheric Environmnet, vol. 40, pp. 3111-3122, 2006.

[17] G. Venetsanos, D. Vlachogiannis, A. Papadopoulos, J. G. Bartzis, and S. Andronopoulos, "Studies on Pollutant Dispersion from Moving Vehicles," Water, Air and Soil Pollution, pp. 325-337, 2002.
[18] M. M. Khan, "Vehicle Combustion Quality Monitoring," in 11th International Conference on Control, Automation, Robotics and Vision (ICARCV) Singapore: IEEE Press, 2010.

[19] K. J. van Wijk, "Image Based Flow Visualization," ACM Transactions on Graphics, vol. 21, 2002.

[20] T. F. Chan and C.-K. Wong, "Total Variation Blind Deconvolution," IEEE Transactions on Image Processing, vol. 7, pp. 370-375, 1998.

[21] S. Szapiel, "Aberration-variance-based formula for calculating pointspread functions: rotationally symmetric aberrations," Applied Optics, vol. 25, pp. 244-251, 1986.

[22] R. M. Haralick, "Digital Step Edges from Zero Crossing of Second Directional Derivatives," IEEE Transactions on Pattern Analysis and Machine Intelligence, vol. 6, pp. 58-68, 1984.

[23] R. C. Gonzalez and P. Wintz, Digital Image Processing. Reading, MA: Addison-Wesley Publishing Co., 1987. 